\title{
Open and Calm - A randomized controlled trial evaluating a public stress reduction program in Denmark
}

\author{
Christian G. Jensen ${ }^{1 *}$, Jon Lansner ${ }^{1}$, Anders Petersen², Signe A. Vangkilde², Signe P. Ringkøbing ${ }^{1}$, Vibe G. Frokjaer ${ }^{1}$,
} Dea Adamsen', Gitte M. Knudsen', John W. Denninger ${ }^{3}$ and Steen G. Hasselbalch ${ }^{1,4}$

\begin{abstract}
Background: Prolonged psychological stress is a risk factor for illness and constitutes an increasing public health challenge creating a need to develop public interventions specifically targeting stress and promoting mental health. The present randomized controlled trial evaluated health effects of a novel program: Relaxation-Response-based Mental Health Promotion (RR-MHP).

Methods: The multimodal, meditation-based course was publicly entitled "Open and Calm" (OC) because it consistently trained relaxed and receptive ("Open") attention, and consciously non-intervening ("Calm") witnessing, in two standardized formats (individual or group) over nine weeks. Seventy-two participants who complained to their general practitioner about reduced daily functioning due to prolonged stress or who responded to an online health survey on stress were randomly assigned to OC formats or treatment as usual, involving e.g., unstandardized consultations with their general practitioner. Outcomes included perceived stress, depressive symptoms, quality of life, sleep disturbances, mental health, salivary cortisol, and visual perception. Control variables comprised a genetic stress-resiliency factor (serotonergic transporter genotype; 5-HTTLPR), demographics, personality, self-reported inattentiveness, and course format.
\end{abstract}

Results: Intent-to-treat analyses showed significantly larger improvements in OC than in controls on all outcomes. Treatment effects on self-reported outcomes were sustained after 3 months and were not related to age, gender, education, or course format. The dropout rate was only $6 \%$.

Conclusions: The standardized OC program reduced stress and improved mental health for a period of 3 months. Further testing of the OC program for public mental health promotion and reduction of stress-related illnesses is therefore warranted. A larger implementation is in progress.

Trial registration: ClinicalTrials.gov:: NCT02140307. Registered May 142014.

Keywords: Stress reduction, Mental health promotion, Meditation, Cortisol, Attention

\section{Background}

Public health sectors in modernized countries are burdened by growing reports of prolonged, psychosocial stress. Otherwise healthy individuals experience that the demands of the environment (most often their occupation) exceed their available resources to a degree that

\footnotetext{
* Correspondence: cgj@cfps.dk

${ }^{1}$ Neurobiology Research Unit (NRU) and Center for Integrated Molecular Brain Imaging (Cimbi), The Neuroscience Centre, Rigshospitalet and University of Copenhagen, Juliane Maries Vej 28, 3rd floor, 2100 Copenhagen OE, Denmark

Full list of author information is available at the end of the article
}

disrupts their daily functioning by way of e.g., concentration problems, irritability, anxiousness, depressive symptoms, fatigue, or bodily pain. About a fourth of North Americans regularly experience high levels of stress [2]. In Denmark, such estimates increased from $6 \%$ in 1987, to $9 \%$ in 2005, and $15 \%$ in 2012 [22, 38]. Prolonged stress is associated with impairments of the cardiovascular, immune, metabolic and nervous systems [56]. For example, long-term psychosocial stress is related to significant increases in neurological inflammatory processes 
[51], and with increased risk for depression [37]. Recent research also connects stress to sleep disturbances [66].

For these reasons, health agencies have underlined a public need for evidence-based programs specifically targeting psychosocial stress and promoting stress resiliency [84]. This was also governmentally reinforced in Denmark [7]. Unfortunately, only about $5 \%$ of Danish health research concerns public health [36].

Therefore, we developed a program designed for stressed, but otherwise healthy adults to reduce stress and promote mental health and resiliency. Reviews have documented that meditation-based multimodal programs reliably reduce stress in healthy samples [21, 33, 68]. However, meditative programs are generally modeled on complex philosophical-religious systems and not academic theories [68]. As an exception, the so-called Relaxation Response (RR) research tradition lead by Herbert Benson and colleagues has through four decades provided empirical evidence supporting that a few core methodological commonalities are evident across many contemplative traditions', and that regular practicing of these techniques is sufficient for eliciting physiological stress reduction and for improving overall health [65]. In targeting a broad demographic group, and since we aimed to develop a theoretically driven and methodologically consistent and well-defined program, we selected RR-based meditation. For the same reasons, we structured the course content according to the wellestablished body-psycho-social understanding of stress (e.g., [56]). Finally, a novel, cognitive framework model termed "Open and Calm" (OC) was used every week to integrate the meditative, bodily, cognitive, and social practices.

Our primary hypotheses were that $\mathrm{OC}$ would reduce self-reported perceived stress as well as physiological stress as measured by cortisol secretion upon awakening [30]. Based on longitudinal studies suggesting that a blunted HPA-axis response to awakening develops with prolonged distress over time [6] and on several studies associating burnout with blunted HPA-axis reactivity $[43,54,59,67]$, we held the secondary hypothesis that intervention participants with blunted baseline cortisol secretion curves upon awakening would exhibit a reestablishment of HPA-axis reactivity. Oppositely, stressed intervention participants with non-blunted cortisol reactivity were predicted to decrease their cortisol awakening response relative to controls. However, HPA-axis dysregulation in relation to prolonged stress and burnout is complex and not fully understood [25]. In further secondary hypotheses, we stated that OC would improve self-reported mental health, quality of life, symptoms of depression, and sleep disturbances, as well as visual attention, as argued by theories of mechanisms of change in meditation $[4,14]$.
It was also theoretically important to investigate several potential treatment effect moderators: First, carriers of $\mathrm{S}$ and LG alleles in the serotonin transporter-linked polymorphic region (5-HTTLPR) of the SCL6A4 gene [16] show increased risk for depression after severe stress in most population studies [46], as well as increased cortisol response to stressors [20, 60]. Second, the personality trait "harm avoidance", reflecting a proclivity to repress stressful stimuli, may decrease stress resiliency, while increased "self-directedness", reflecting overall top-down self-regulation abilities, may promote stress resiliency [72]. Third, variables such as age, gender, and education are recommendable covariates in public health promotion to evaluate the demographic applicability of the intervention [45].

\section{Method}

\section{Participants}

Participants were stressed, but otherwise healthy, Danish adults (65\% women) aged $18-59$ years (Mean $=42$ years, Standard Deviation $=9$ years, interquartile range: $36-$ 48). Participants seldom reported to have no professional education, and relatively often to have longer professional educations, compared with the Copenhagen adult population at the time [70] (\% of sample/\% of population: no professional education: $8 \% / 33 \%$; apprenticeship: $24 \% / 22 \%$; $1-3$ years: $14 \% / 5 \%$; bachelor degree or 3-4 years: $24 \% / 22 \%$; 4 years: $31 \% / 19 \%)$. All were Caucasian. The majority (92 \%) never meditated regularly (>2 times/week for $>1$ month) before. Additional file 1: Table S1 provides more detailed sample characteristics. The inclusion criteria were the age 18-59 years, fluency in Danish, and subjective report of reduced daily functioning due to stress for more than one month. This was evaluated in a 1-hr personal inclusion interview (Fig. 1 shows the participant flow). The main exclusion criteria were current treatment for any illness; $>1$ diagnosed or treated ICD-10 mood disorder (F30-39) or any other ICD-10 disorder for adults within three years; Hamilton Depression Rating Scale score $>20$ at the inclusion interview; recreational drug use $>24$ times per year or $>50$ times in the lifetime; Body-Mass-Index (BMI) $>30$ (due to exploratory psycho-physiological measurements), and medication use affecting the brain or cortisol, such as selective serotonin reuptake inhibitors or corticosteoroid medications.

\section{Procedures}

The present Clinical Registered Trial (Clinicaltrials.gov ID: NCT02140307), approved by the Danish Ethics Committee (H-3-2012-092), recruited volunteers through 20 General Practitioners (GP) and an online medical recruitment company. Figure 1 shows participant flow and retest rates. Participants provided informed consent. Stratifying 


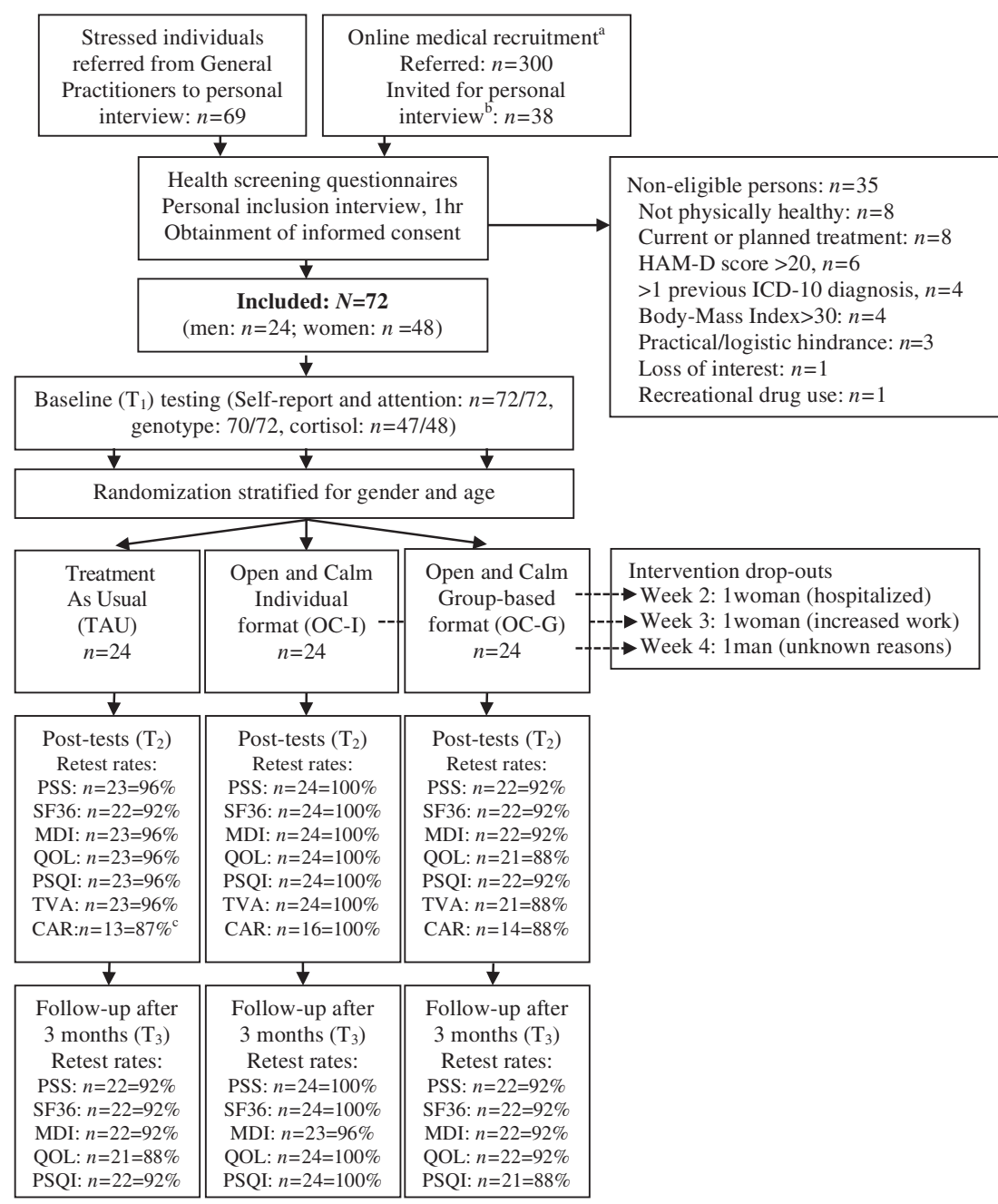

Notes. HAM-D=Hamilton Depression Rating Scale 17 items. PSS=Perceived Stress Scale. SF36=Short-Form Health Survey Mental Health Component Summary Score. MDI=Major Depression Inventory. QOL=Quality of Life. PSQI=Pittsburgh Sleep Quality Index. TVA=Theory of Visual Attention test. CAR=Cortisol Awakening Response test. ${ }^{\mathrm{a}}$. Online invitations were issued by the professional recruitment company within public health, Medicollect.

${ }^{\mathrm{b}}$.Interviews were conducted by the first author (XX), a clinical neuropsychologist and experienced meditator. ${ }^{\mathrm{c}}$. The retest ratio is $87 \%$ ( $n=13 / 15)$ since only 15 cortisol sets from TAU participants were received before randomization.

Fig. 1 Participant flow in the Open and Calm Randomized Controlled Trial

for age and gender, the last author SGH, who had no participant contact, block-randomized three consecutively enrolled cohorts of $n=24$ to intervention in individual format, group-format, or treatment as usual (TAU), involving e.g., extra GP visits, or stress leave. Groups were randomized with a ratio of 1:1:1 using www.random.org. An a priori power calculation in G-power [29] revealed a required $N=54$ (power $=.95$, three groups, three measurements [pre, post, follow-up], expected effect $f=0.25$, sphericity correction $=1$ ). This power analysis did not specify the number of expected covariates, since the theoretical knowledge on effect moderators in meditation-based stress reduction programs is very limited [35], especially for healthy samples [68]. A medium effect size after meditation-based stress reduction programs is commonly found on self-report outcomes [68]. Effect sizes for behavioral and physiological outcomes vary more in studies of meditation-based stress reduction [55], but we have previously demonstrated medium-large effects of mindfulnessbased stress reduction (MBSR; [44]) on the presently investigated physiological and perceptual tests and outcomes [42]. Expecting 15-30 \% dropout [64], $N=72$ were therefore recruited.

Danish, validated self-report instruments were completed online at home. Double-blinded baseline data $\left(\mathrm{T}_{1}\right.$, Jan.-Mar., 2013) were obtained before randomization 
(Fig. 1). To increase validity, scales were completed both at 4 weeks and 2 weeks pre-intervention and 2 weeks and 4 weeks post-intervention (Apr.-Jun, 2013). $\mathrm{T}_{1}$ and $\mathrm{T}_{2}$ scores reflect the average of each pair of completions, as recommended [80]. Cortisol (for financial reasons only collected for the first $n=48$ ) and attention were tested within 2 weeks before and after the intervention period by researchers blinded to participant status at $T_{2}$. Follow-up 3 months after the intervention $\left(\mathrm{T}_{3}\right.$, Oct.Dec., 2013) included self-report. Participants were not contacted during the follow-up period itself.

\section{The intervention}

The "Open and Calm" (OC) program was based on the Relaxation Response (RR) tradition [65], which has for decades empirically supported that many meditative techniques elicit the same physiological, parasympathetic $\mathrm{RR}$, involving e.g., lowered heart rate, blood pressure, and respiration rate. RR theory proposes that the core methods across meditative techniques necessary for eliciting the RR are: (a) the continuous returning of attention to a meaningful focus, i.e., focused attention training, and (b) the non-reactive or contemplative witnessing of ongoing experience. The OC meditation focused on these aspects and termed them "Open" (relaxed and receptive attentiveness) and "Calm" (nonintervening witnessing).

The course structure was modeled on the wellestablished overall understanding that bodily (biological), psychological, and social factors interact in stress, stress resiliency, and health, focusing each week on working with either the body, the mind, or social relationships, in a cyclic fashion. Meditation was trained every week; bodily, cognitive, and social practices followed the weekly themes. Importantly, all practices focused on training the $\mathrm{OC}$ states (e.g., Open attention toward the breath, an emotion, or another person) and were theoretically integrated by a core OC cognitive framework model.

The OC program is standardized [40] and was offered in two formats: The group format (OC-G) involved weekly 2.5 -hr group sessions ( $\mathrm{n}=8$ per group) and two optional 1.5-hr personal sessions. The individual format (OC-I) involved personal, weekly $1.5-\mathrm{hr}$ sessions. Formats used identical materials, e.g., a 120-page course book [39], online materials, 1-2 daily meditations of 1020 min following audio files, and frequent "mini-meditations" of 1-2 $\mathrm{min}$.

\section{Measures}

\section{Control variables}

\section{Demographics and life style}

Demographic factors investigated as covariates included age, gender, education, and occupational status (employed/unemployed). Life style variables included previous meditation experience, alcohol consumption, tobacco use, and BMI.

Genotype Saliva was collected in DNA Genotek tubes (Ottawa, Canada) and frozen at -80 degrees Celsius until analyzed. Polymerase chain reaction (PCR) was used to amplify the 5-HTTLPR and two oligonucleotide primers [83] to generate allele-specific fragments: short (S) allele $469 \mathrm{bp}$ and long (L) allele $512 \mathrm{bp}$. PCR was performed in a GeneAmp PCR System 9700 (Applied Biosystems MspI). The genotype covariate quantified the efficiency of 5 - HT reuptake: $0=\mathrm{SS} / \mathrm{SLG}, n=14$; $1=$ SLA/LGLA, $n=41 ; 2=$ LALA, $n=15$; missing: $n=2$.

Personality From Temperament and Character Inventory (TCI) [23] the personality trait Harm Avoidance (TCI-HA) evaluated the proclivity to avoid novelty, nonreward and punishment. The trait Self-Directedness (TCI-SD) measured executive, self-regulation and adaption. TCI-HA and TCI-SD were recommended as screening tools in public health studies of stress [72]. Both factors were internally consistent, Cronbach's $\alpha \mathrm{s} \geq .84$.

Stressful life events Stressful Life Events (SLE; [47]) was used to investigate SLE within the past year and the lifetime (e.g., assault, job loss, serious illness, loss of a confidant).

Attentional instability Mindful Attention Awareness Scale (MAAS; [11]) evaluated attentional instability at baseline via 15 items and was internally consistent, $\alpha=.88$. The Danish translation of the MAAS has been psychometrically validated [41].

Test motivation At each cognitive test, participants rated how motivated they were to comply with the task on a 7-point Likert-scale from 0 (not at all motivated) to 6 (very motivated).

Course attendance Course length or the number of treatment days $[17,68,81]$, as well as the degree of compliance with meditation pratices [74, 79] have not shown consistent relationships with effects of mindfulnessbased programs for stress reduction or health promotion. Similarly, a comprehensive review of an RR-based stress reduction program found that treatment effects on stress-related outcomes (anxiety and hostility) were not related to pre-post changes in weekly meditation practices [18]. To minimize participant burden and lower dropout, compliance was presently only quantified as the number of attended $\mathrm{OC}$ sessions. 


\section{Outcome variables \\ Perceived stress}

Cohen's Perceived Stress Scale (PSS; [24]) comprise 10 items of stress-related experiences rated from 0 (never) to 4 (very often) for their frequency during the past two weeks, providing an overall score. The PSS was always internally consistent, $\alpha \mathrm{s} \geq .82$.

Mental health Short-Form Health Survey-36 (SF-36; [82]) measures eight health dimensions: 1) physical function, 2) physical role limitations, 3) bodily pain, 4) general health, 5) emotional function, 6) vitality, 7) emotional role limitations, and 8) mental health. Each dimension is scored from 0 (poor) to 100 (best possible). The Mental Component Summary score (SF-36-MCS) was based on weighting of all dimensions [5]. At all times, $\alpha$ was $\geq .71$.

Depressive symptoms Major Depression Inventory (MDI; [3]) involve ratings of the frequency of the ten ICD-10 depressive symptoms during the past two weeks $(0=$ not at all, $5=$ all of the time $)$. The total MDI was investigated and was always internally consistent, $\alpha \mathrm{s}>.83$.

Quality of life The 5-items Quality of Life (QOL) developed by WHO assesses quality of life through positive affect and vitality. The Danish QOL has high validity and QOL scores $<50$ is a risk marker for depression [31]. QOL was internally consistent, all present $\alpha \mathrm{s}>.81$.

Sleep disturbances Pittsburgh Sleep Quality Index (PSQI; [15] indexes sleep disturbances during the past month via 19 items. On the examined PSQI Global, scores $>5$ indicate increased risk for depression. Consistency was mostly satisfactory, $\alpha \mathrm{s}: \mathrm{T}_{1}=.61 ; \mathrm{T}_{2}=.77$; $\mathrm{T}_{3}=.69$.

Physiological stress The cortisol awakening response (CAR) reflects hypothalamic-pituitary-adrenal (HPA) axis activity [30]. After written and verbal instructions and training, participants performed home-samplings of saliva in Salivette tubes (Sarstedt, Neubringen, Germany). Sample 1 was taken immediately upon awakening, and samples $2-5$ every $15 \mathrm{~min}$ for the subsequent hour. Participants registered the time of awakening and of each sampling. Within $48 \mathrm{hrs}$ samples were centrifuged and stored at -80 degrees Celsius. The entire batch was analyzed in one step using electrochemiluminescent immunoassay (Cobas equipment, Roche, Germany). Our outcomes were the Area Under the Curve with respect to ground $\left(\mathrm{AUC}_{\mathrm{G}}\right)$, representing the total magnitude of cortisol secretion; and the Area Under the Curve with respect to increase from awakening levels $\left(\mathrm{AUC}_{\mathrm{I}}\right)$, reflecting the HPA axis' cortisol response to awakening [30]. Participants with symptoms of burnout at $\mathrm{T}_{1}$ (blunted $\mathrm{CAR}\left[\mathrm{AUC}_{\mathrm{I}}\right]$ curves) were analyzed separately. Blunted $\mathrm{T}_{1}$ CAR curves were identified by inspection of individual curves by two researchers (anonymized, anonymized) blinded to participant group status.

Visual attention The computational Theory of Visual Attention (TVA; [12]) framework quantifies functions of visual attention using accuracy-based testing. The TVAbased test used here (ad modum [77]) comprised two practice blocks and three test blocks of 30 trials presenting six red letters on a computer screen. The letter display durations were varied systematically $(20-200 \mathrm{~ms})$, and terminated by pattern masks $(500 \mathrm{~ms})$ before participants made an unspeeded report of letters they were "fairly certain" of having seen. In cognitive test rooms, participants were instructed to refrain from pure guessing and to aim for an accuracy of $80-90 \%$. They were informed about their accuracy after each block. Three parameters of attention were extracted by mathematical modeling [28]: The storage capacity of visual short-term memory ( $K ; 5$ degrees of freedom $[\mathrm{df}]$ ), the speed of visual processing $(C ; 1 \mathrm{df})$, and the threshold for conscious visual perception $\left(t_{0} ; 1 \mathrm{df}\right)$. Since meditation may specifically improve visual perceptual thresholds $[42,52], t_{0}$ was our visual attention outcome, while $K$ and $C$ analyses were exploratory.

\section{Statistical analyses}

Intent-To-Treat (ITT) models were applied, replacing missing $T_{2}$ or $T_{3}$ scores with $T_{1}$ or $T_{2}$ scores, respectively. Group differences in outcome changes were investigated in two-way repeated measures ANCOVAs using Time ( $\left.\mathrm{T}_{1} / \mathrm{T}_{2} / \mathrm{T}_{3}\right)$ and Group (e.g., OC/ TAU controls) as independent variables. These main analyses adjusted for covariates (see 'Control Variables') that correlated with $(p<.05)$ outcome change scores $\left(\mathrm{T}_{1}-\mathrm{T}_{3}\right)$ within the compared groups. Adjustment was carried out by including Time $\times$ Group $\times$ Covariate-interactions in the models. Continuous covariates were centered before entering the models $[1,73]$. Significant Time $\times$ Group $\times$ Covariate-interactions were interpreted as indications of a potential effect-moderating role for the covariate $[50,76]$. These interpretations were based on post hoc visual plots and correlation tests as described in the Results section.

Candidate covariates were selected based on theories and studies of mechanisms of change in meditationbased stress reduction, while the actual inclusion of covariates in the final analyses was data-driven. This strategy was applied because $\mathrm{OC}$ is a new program and because theories on moderators and mediators of change in meditation-based programs are preliminary and 
sparse [35, 48]. This is especially the case for studies healthy samples, which have "largely been conducted in a rather atheoretical manner" ([68], p. 1161). To promote progress in this area, we discuss potential effect moderators, but the present RCT focused on investigating the effectiveness of OC compared to TAU.

We explored different analytic approaches by also including covariates that were associated with $(p<.05)$ outcome scores at baseline and by examining $T_{1}-T_{2}$ change scores' associations with candidate covariates. We also tried excluding all scores $>3.0 \mathrm{SD}$ from group means $(<2 \%$ in all analyses). These analytic variations did not change any Time ${ }^{\perp}$ Group results significantly. In the final analyses, one score was excluded, being a $\mathrm{T}_{2} t_{0}$-value $\left(0.7 \%\right.$; replaced with the $\mathrm{T}_{1} t_{0}$-value) of inadequate data quality. All $p$-values were Bonferroni-Holmcorrected for the number of group comparisons within each outcome type (self-report/cortisol/attention). The analysis of Time ${ }^{\perp}$ Group interactions on depressive symptoms violated the assumption of sphericity (Mauchly's $W=0.87, p<.05$ ) and was thus Huyn-Feldtcorrected.

OC format was not expected to affect intervention effects $[9,53,81]$, but this was investigated in an initial OC-I vs. OC-G comparison. If formats did not differ $(p<.05)$, the collapsed OC was then compared to TAU controls. If formats did differ, each format was to be compared to controls in turn. A multivariate analysis of covariance (MANCOVA) examined whether gender, age (median split), or education ( $3 d f$ ) affected long-term $\left(\mathrm{T}_{1}-\mathrm{T}_{3}\right)$ change scores across all selfreport scales in OC. The MANCOVA was designed to test the socioeconomic applicability of $\mathrm{OC}$ and therefore did not include other candidate effect moderators, such as genotype or personality. To strengthen our interpretation, we followed up the MANCOVA with a series of zeroorder post hoc correlations testing associations between each demographic factor (age, gender, and education) and short-term $\left(\mathrm{T}_{1}-\mathrm{T}_{2}\right)$ as well as long-term $\left(\mathrm{T}_{1}-\mathrm{T}_{3}\right)$ outcome changes, respectively.

Effect sizes were expressed with Cohen's $d$ (group differences and pre-post within group effects corrected for dependence among means ad modum [58]; formula 8), Pearsons $r$ or Spearman's rho $(\rho)$ (variable associations), or partial eta-squared, $\eta_{p}^{2}$, (Time $\times$ Group effects and Time $\times$ Group $\times$ covariate interactions). MDI and PSQI data were skewed and $\log ^{10}$-transformed, yielding normal distributions. Questionnaires' internal consistency was assessed with Cronbach's alpha, $\alpha$. We pre-defined $\mathrm{AUC}_{\mathrm{G}}$ and PSS as primary outcomes in the trial protocol. Similarly, we specified $\mathrm{AUC}_{\mathrm{I}}$, SF-36-MCS, MDI, QOL, PSQI, and $t_{0}$ as secondary outcomes. Analyses were carried out in SPSS (IBM, vs. 20.0) and Microsoft Excel 2011.

\section{Results}

\section{Course attendance}

OC had a $94 \%(n=45 / 48)$ completion rate. The three dropouts did not differ from other participants on any baseline characteristics. In total, group participants attended more sessions $(\operatorname{mean}[\mathrm{M}]=8.3, \quad S D=2.7)$ than individual format participants $(\mathrm{M}=6.7, S D=2.0)$ $(p=.020)$, but required less $(\mathrm{M}=3.9 \mathrm{hrs}, \quad S D=1.7)$ professional contact hours per participant $(\mathrm{M}=$ $10.0 \mathrm{hrs}, S D=3.0$ ), a ratio of 2.56 . Session attendance rates were unrelated to outcome changes unless otherwise is stated.

\section{Self-report}

Intervention format did not affect changes in the primary self-report outcome of perceived stress, PSS, $p=.13$ ), or any secondary self-report changes, $p \mathrm{~s}>.1$ (uncorrected $p s \geq .06$; Additional file 2: Panel S1). The total intervention group improved significantly more than TAU controls on PSS, $p<.0001$. Similarly, OC improved significantly more than TAU on all secondary self-report scales, $p$ s $<.005$ (Table 1 and Fig. 2). Effects were sustained or significantly improved on all scales during follow-up and OC differed significantly from controls on all scales at $\mathrm{T}_{3}, p \mathrm{~s}<.02$. OC increased above the quality of life risk marker for depression; controls did not (Fig. 2, d). OC decreased slightly below the sleep disturbances risk marker for depression; controls did not (Fig. 2 , e). The MANCOVA showed no effect of age, gender, or education across self-report effects for $\mathrm{OC}, p>.2$. In line with this, age, gender, and education were unrelated with both short-term $\left(\mathrm{T}_{1}-\mathrm{T}_{2}\right)$ and longterm $\left(\mathrm{T}_{1}-\mathrm{T}_{3}\right)$ changes, respectively, on all self-report outcomes, $r s \leq .21, p s>.17$. (See further details on potential effect moderators in Additional file 3: Supplementary findings-potential effect moderators).

\section{Physiological stress}

Cortisol outcomes were normally distributed in all groups at all time points, $p \mathrm{~s}>.15$ (Shapiro-Wilk). OC-I and $\mathrm{OC}-\mathrm{G}$ did not differ at any time point or from prepost treatment, $p s>.09$ (uncorrected). We then compared all OC participants to all TAU controls. In these primary analyses, the two groups did not differ on any cortisol outcomes at baseline, post-treatment, or in changes from pre-post (Additional file 4: Table S2). However, our secondary hypothesis stated that baseline CAR profile (blunted/non-blunted $\mathrm{T}_{1} \mathrm{CAR}$ ) would affect the directionality of CAR changes for OC participants. In support of this, OC participants with a non-blunted $\mathrm{T}_{1}$ CAR decreased significantly on $\mathrm{AUC}_{\mathrm{G}}, p=.018$, $d=-0.59$ (Additional file 5: Panel S2; Additional file 4: Table S2). This decrease in OC was significantly larger than in non-blunted controls, $p=.030$ (corrected), $\eta_{p}^{2}=.24$ (Fig. 3; Additional file 4: Table S2). Since OC 
Table 1 Treatment effects on self-report outcomes

\begin{tabular}{|c|c|c|c|c|c|c|c|c|c|c|}
\hline \multirow[t]{2}{*}{ Outcome } & \multicolumn{2}{|c|}{ Open and Calm (OC) } & \multicolumn{2}{|c|}{ Treatment as Usual (TAU) } & \multirow{2}{*}{$\begin{array}{l}\text { OC vs. TAU } \\
\text { d (between) }\end{array}$} & \multicolumn{3}{|c|}{ OC vs. TAU change } & \multirow[b]{2}{*}{$\mathrm{p}$} & \multirow[b]{2}{*}{ Covariates } \\
\hline & $\bar{M}(S D)$ & $\mathrm{d}$ (within) & $\mathrm{M}(\mathrm{SD})$ & $\overline{d \text { (within) }}$ & & $p$ & $\mathrm{~F}$ & $\overline{\eta_{p}^{2} \text { (between) }}$ & & \\
\hline \multicolumn{11}{|l|}{ Perceived Stress (PSS) } \\
\hline Pre-treatment $\left(T_{1}\right)$ & $18.75(6.48)$ & & $18.22(4.01)$ & & 0.09 & .718 & 12.96 & $.16^{* * * *}$ & $<.0001$ & \\
\hline Post-treatment ${ }^{\mathrm{b}}\left(\mathrm{T}_{2}\right)$ & $12.88(7.31)$ & $0.92^{* * *}$ & $17.33(3.51)$ & 0.22 & $0.71^{* *}$ & .012 & & & & \multirow{3}{*}{$\begin{array}{l}\text { Employment } \\
\text { TCl-HA }\end{array}$} \\
\hline Follow-up ${ }^{c}\left(T_{3}\right)$ & $11.64(6.26)$ & 0.24 & $16.77(3.83)$ & 0.25 & $0.93^{* * *}$ & .001 & & & & \\
\hline Pre-treatment-Follow-up & & $1.30^{* * *}$ & & $0.39^{*}$ & & & & & & \\
\hline \multicolumn{11}{|l|}{ Mental Health (SF-36-MCS) } \\
\hline Pre-treatment $\left(\mathrm{T}_{1}\right)$ & $47.24(26.05)$ & & $55.06(17.26)$ & & 0.20 & .299 & 4.97 & $.13^{* *}$ & $=.0018$ & \\
\hline Post-treatment ${ }^{\mathrm{b}}\left(\mathrm{T}_{2}\right)$ & $51.22(25.17)$ & 0.21 & $52.95(19.26)$ & -0.18 & 0.08 & .971 & & & & Age \\
\hline Follow-up ${ }^{c}\left(T_{3}\right)$ & $67.09(17.57)$ & $0.89^{* * *}$ & $57.73(16.38)$ & 0.30 & $0.55^{*}$ & .012 & & & & \\
\hline Pre-treatment_Follow-up & & $0.99 * * *$ & & 0.15 & & & & & & \\
\hline \multicolumn{11}{|l|}{ Depression (MDI) } \\
\hline Pre-treatment $\left(\mathrm{T}_{1}\right)$ & $16.98(8.67)$ & & $15.75(7.10)$ & & 0.15 & .551 & 8.01 & $.11^{* *}$ & $=.002$ & \\
\hline Post-treatment ${ }^{\mathrm{b}}\left(\mathrm{T}_{2}\right)$ & $10.04(8.65)$ & $0.91 * * *$ & $13.27(5.97)$ & 0.36 & $-0.42^{*}$ & .044 & & & & MAAS \\
\hline Follow-up ${ }^{c}\left(T_{3}\right)$ & $8.04(6.01)$ & 0.51 & $12.42(6.02)$ & 0.22 & $-0.74^{* *}$ & .006 & & & & \\
\hline Pre-treatment-Follow-up & & $1.44^{* * *}$ & & $0.60^{*}$ & & & & & & \\
\hline \multicolumn{11}{|l|}{ Quality of Life (WHO-5) } \\
\hline Pre-treatment $\left(T_{1}\right)$ & $46.88(17.32)$ & & $48.67(15.72)$ & & -0.11 & .671 & 5.71 & $.08^{* *}$ & .004 & \\
\hline Post-treatment $^{\mathrm{b}}\left(\mathrm{T}_{2}\right)$ & $62.04(19.84)$ & $1.01^{* * *}$ & $53.92(14.54)$ & 0.31 & 0.45 & .080 & & & & BMl \\
\hline Follow-up ${ }^{c}\left(T_{3}\right)$ & $65.75(16.44)$ & 0.23 & $55.67(15.19)$ & 0.10 & $0.64^{* *}$ & .014 & & & & TCl-HA \\
\hline Pre-treatment-Follow-up & & $1.06^{* * *}$ & & 0.39 & & & & & & \\
\hline \multicolumn{11}{|l|}{ Sleep Quality (PSQI) } \\
\hline Pre-treatment $\left(\mathrm{T}_{1}\right)$ & $6.97(2.49)$ & & $6.67(2.81)$ & & 0.12 & .531 & 11.27 & $.14^{* * *}$ & $=.0001$ & \\
\hline Post-treatment ${ }^{b}\left(T_{2}\right)$ & $5.43(3.63)$ & $0.47^{* * *}$ & $5.92(2.73)$ & 0.25 & -0.15 & .254 & & & & Smoking \\
\hline Follow-up ${ }^{c}\left(T_{3}\right)$ & $4.96(2.93)$ & 0.22 & $6.63(3.16)$ & -0.22 & $-.56^{*}$ & .017 & & & & \\
\hline Pre-treatment-Follow-up & & $0.73^{* * *}$ & & 0.01 & & & & & & \\
\hline
\end{tabular}

Notes. ${ }^{*} . p<.05 .{ }^{* *} . p<.01 .{ }^{* * *} . p<.001$. All $p$-values are two-tailed , Bonferroni-Holm corrected, and based on intent-to-treat-analyses (OC: $n=48 ;$ TAU: $\left.n=24\right)$. The OC vs. TAU change-models adjusted for relevant biopsychosocial covariates (listed in the column "Covariates") as well as for Time Group Covariate-interactions.

${ }^{\text {a }}$. Effect sizes indicate pre-treatment-post-treatment-follow-up Time Group effects. ${ }^{b}$. Within-group effect sizes indicate pre-treatment-post-treatment effects.

c. Within-group effect sizes indicate post-treatment-follow-up effects. MAAS = Mindful Attention Awareness Scale. MDI=Major Depression Inventory. PSQI=Pittsburgh Sleep Quality Index. PSS=Cohen's Perceived Stress Scale. SF-36-MCS=Short Form Health Survey-36-Mental Component Summary. TCI-HA = Temperament and Character Inventory - Harm-avoidance. Smoking = Smokers was defined as participants who smoked daily, while other participants were defined as non-smokers

showed non-significantly higher baseline cortisol $\left(\mathrm{AUC}_{\mathrm{G}}\right)$ levels, we conducted a post hoc ANCOVA also controlling for baseline $\mathrm{AUC}_{\mathrm{G}}$, and still controlling for TCI-HA, age, and the three interaction terms. This test reaffirmed that the treatment group decreased significantly more than controls (Time $\times$ Group interaction) $F(1,20)=5.09, p=.035, \eta_{p}^{2}=.20$. Group changes for $\mathrm{AUC}_{\mathrm{I}}$ did not differ, but only $\mathrm{OC}$ decreased significantly, $p=.018, d=-0.76$ (Additional file 5: Panel S2, a). Blunted baseline CAR was identified for $n=18$ in $\mathrm{OC}$, and $n=2$ in TAU. Group comparisons were therefore not meaningful for these $\mathrm{AUC}_{\mathrm{I}}$ analyses. As we hypothesized, CAR-blunted OC participants showed a significantly increased $\mathrm{AUC}_{\mathrm{I}}, p=.015, d=0.88$ (Additional file 5: Panel S2, b). This effect suggested a healthy reestablishment of
HPA-axis reactivity to awakening. This interpretation was supported by inspection of visual plots suggesting that the increase in $\mathrm{AUC}_{\mathrm{I}}$ for CAR-blunted $\mathrm{OC}$ participants was due to a lowered cortisol awakening level (sample 1 taken immediately upon awakening), followed by a more dynamic HPA-axis response to awakening (Additional file 5: Panel S2, c-d).

\section{Visual attention}

Intervention format did not affect changes in the perceptual threshold, $t_{0}, p>.6$ (Additional file 2: Panel S1, f). The total intervention group improved significantly more than controls on $t_{0}\left(p<.05, \eta_{p}^{2}=.056\right)$ due to significant improvement in $\mathrm{OC}$ and no change in controls (Additional file 4: Table S2). A post hoc ANCOVA controlling for $t_{0}$ 


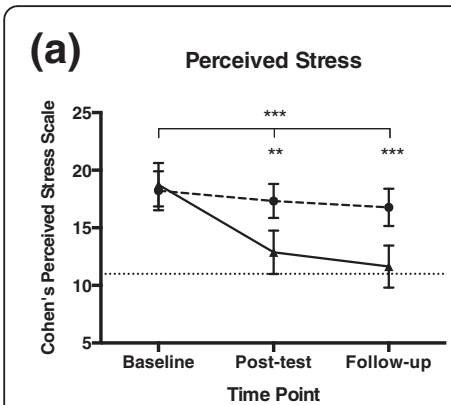

(d)

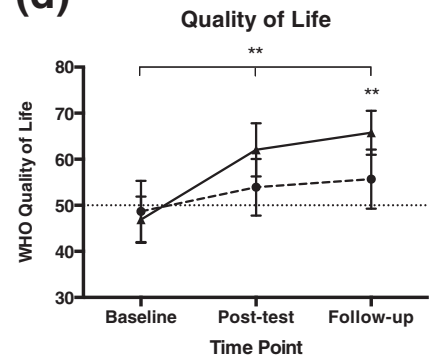

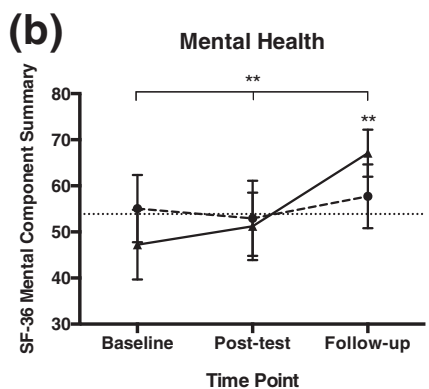

(e)

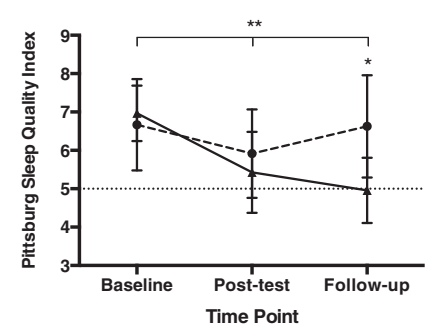

(c) Symptoms of Depression

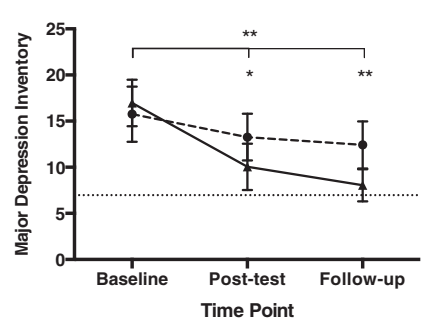

$\mp \mathrm{OC}$

Notes. ${ }^{*} . p<.05 .^{* *} . p<.01 .^{* \star *} . p<.001 . p$-values are two-tailed, corrected for multiple tests (Bonferroni-Holm), and based on intent-to-treat-analyses (Open and Calm [OC] $N=48$. Treatment As Usual [TAU] $N=24$ ) after adjustment for relevant biological, socioeconomic, and psychological trait variables. Asterisks (*) above horizontal lines represent $p$-values of Time*Group effects, while asterisks or $p$-values above error bars represent $p$-values of between-group comparisons (Table 2). Error bars represent $95 \% \mathrm{Cl}$ of the mean. (a). The dotted line represents the mean among a national region-stratified random sample of >21,000 Danish adults (Stigsdotter et al., 2010). (b) The dotted line represents the age-adjusted Danish norm for the SF36-Mental Health Component (Bjømer et al., 1997)(c). The dotted line represents the Danish norm (Olsen et al., 2004). (d) Scores below the dotted line represent a risk marker for depression (Folker \& Folker, 2008). As seen, the $95 \% \mathrm{Cl}$ still contains this cut-off for TAU, but not for OC. (e) Scores above the dotted line represent a risk marker for depression (Buysse et al., 1989). TAU remains at increased risk at all time points. Specifically, $67 \%$ of OC and $63 \%$ of TAU were at increased risk at baseline. At follow-up, this was still found for $63 \%$ of TAU, but only $35 \%$ of OC.

Fig. 2 Group comparisons on self-report outcomes

at baseline, as well as for the other baseline covariates, still supported a significant Time $\times$ Group interaction, $F(1,64)=5.06, p=.028, \eta_{p}^{2}=.073$. Additionally, higher $\mathrm{OC}$ attendance rates were indicative of larger $t_{0}$ improvement, $\rho=-.33, p=.023$. The exploratory analyses of visual short-term memory capacity, $K$, and processing speed, $C$, showed no significant treatment effects, $p \mathrm{~s}>.2$ (uncorrected). Groups did not differ at any time point or from pre-post treatment in their self-reported motivation to perform the test, $p \mathrm{~s}>.1$.

\section{Discussion}

Experiences of prolonged psychosocial stress is currently not targeted by evidence-based programs in most public health sectors [45]. The present RCT supported that the meditation-based program "Open and Calm" (OC) developed for this purpose decreased participants' perceived stress, depressive symptoms, and sleep disturbances, and increased their self-reported mental health and quality of life significantly more than the Danish health sector's treatment as usual (TAU) for otherwise healthy adults complaining about reduced daily functioning due to prolonged stress. Treatment effects were consistently sustained at 3 months follow-up and $\mathrm{OC}$ participants reported significantly better mental health than TAU controls at follow-up on all self-report scales (Table 1).
According to well-established cut-offs, OC reduced the risk for depression due to poor quality of life (QOL; [31]) and sleep disturbances (PSQI; [15]). OC participants reported follow-up levels corresponding to Danish norms for perceived stress [71], mental health [5], and symptoms of depression [63] (Fig. 2). Control participants showed heightened risk for depression and suboptimal mental health scores throughout the six months study period.

OC improved the included self-report parameters with similar or slightly larger effect sizes (mean self-report $\mathrm{T}_{1}-\mathrm{T}_{3} d=1.10$; mean self-report $\mathrm{T}_{1}-\mathrm{T}_{2} d=0.70$; Table 1 ) than typically found in studies of healthy samples participating in courses based on mindfulness meditation or transcendental meditation, according to meta-analytic reviews ([21]: $d=0.74 ;[34]: d=0.50 ;[68]: d \mathrm{~s}=0.54-$ 0.56). Similarly, the effect from baseline to 3-month follow-up of OC on Cohen's perceived stress scale (PSS; $d=1.30$ ) was larger than a baseline-3-months follow-up analysis on PSS of public stress reduction workshops based on cognitive and/or behavioral therapy ([53], mean $d=0.91)$. Thus, OC seems promising compared with other stress reduction programs. However, the present findings, especially the physiological results, should be interpreted with caution due to the limited sample size compared to meta-analytic reviews. Further and larger OC studies are needed. 


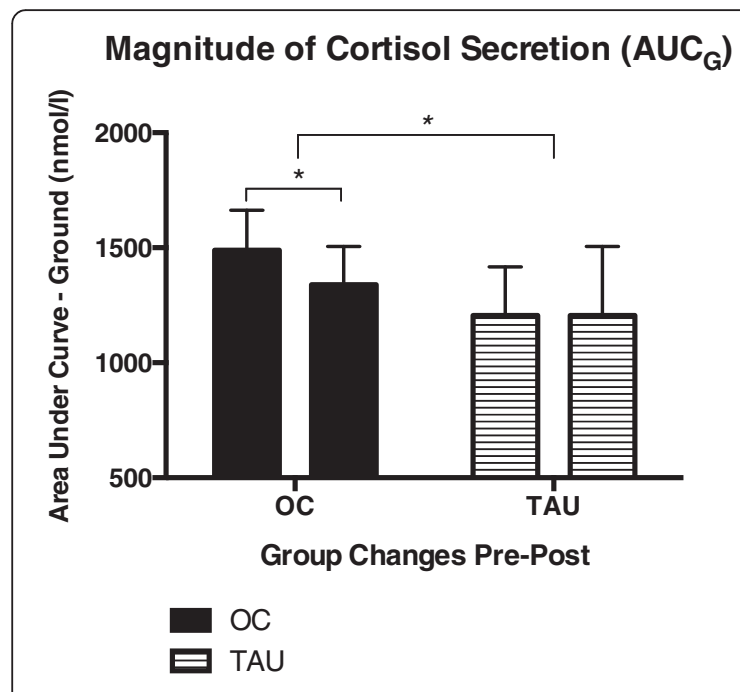

Notes. ${ }^{*} . p<.05 . p$-values are two-tailed and based on intent-to-treat-analyses after adjustment for relevant confounders (age and personality trait harm avoidance; see "Control variables"). Error bars represent $95 \% \mathrm{Cl}$ of the mean. Supplementary Table 2 shows descriptive cortisol data and test statistics. Bars represent Area Under Curve - Ground $\left(A \cup C_{G}\right)$-values for participants with non-blunted baseline cortisol awakening response (CAR): Open and Calm (OC) $n=15$, Treatment As Usual (TAU) $n=13$. The Time ${ }^{*}$ Group effect was still significant after control for baseline $\mathrm{AUC}_{\mathrm{G}}, p=.035$ (see text).

Fig. 3 Changes in cortisol secretion

Physiological stress, in terms of cortisol secretion and HPA-axis dynamics, was also investigated. The primary analyses included all participants and did not show significant changes on any cortisol outcomes in any group (Additional file 4: Table S2). However, based on the potential exhaustion of HPA-axis dynamics after prolonged stress ([6]) and associations between burnout and blunted CAR [43, 54, 59, 67], our secondary analyses separated participants into two subgroups according to their baseline CAR $\left(\mathrm{AUC}_{\mathrm{I}}\right)$ profile. The first subgroup included all participants with an initially present (nonblunted) CAR. Within this group, OC participants decreased significantly on the magnitude of cortisol secretion $\left(\mathrm{AUC}_{\mathrm{G}}\right)$, and also significantly more than in nonblunted TAU controls, even after controlling for relevant covariates and baseline $\mathrm{AUC}_{\mathrm{G}}$ levels. Decreasing circulating levels of cortisol may be important in restoring health and preventing negative consequences of prolonged stress, e.g., because it may prevent neural atrophy in frontal and hippocampal regions, improving topdown regulation of limbic systems, promoting stress resiliency [19]. The stimulated HPA-axis output $\left(\mathrm{AUC}_{\mathrm{I}}\right)$ also decreased significantly in OC participants with nonblunted baseline CAR (Additional file 5: Panel S2, a).
This change may relate to improved stress resiliency, since HPA-axis reactivity has been associated with several risk factors for depression, including 5-HTTLPR genotype [20]. In the present study, 5-HTTLPR genotype was unrelated to any treatment effects. This is contrary to one study indicating stronger physiological stress reduction effects in SS-carriers than SL-carriers in a student sample [61]. More knowledge is needed on genetic effect moderators of stress reduction effects of meditation-based programs in different sample types. Effects of 5-HTTLPR may decrease with age ([75]). In addition, CAR is not a direct measure of individuals' reactivity to everyday stressors (but see $[20,32])$ and effects of 5-HTTLPR-genotype on reactions to stressful stimuli interact with environmental factors [16]. $\mathrm{AUC}_{\mathrm{I}}$ changes did not differ between $\mathrm{OC}$ and TAU. Thus, the main cortisol effect of $\mathrm{OC}$ was a reduction in the magnitude of cortisol secretion for participants with a non-blunted CAR at baseline. In addition, as we hypothesized, $\mathrm{AUC}_{\mathrm{I}}$ increased significantly for OC participants with a blunted baseline CAR. This suggests that HPA-axis dynamics, i.e., HPA axis reactivity to stimulation (awakening), was reestablished (Additional file 5: Panel S2, b). A renormalization of CAR potentially also influenced TAU controls, but we could not evaluate this $\mathrm{AUC}_{\mathrm{I}}$ change statistically with only $n=2$ blunted TAU controls (Additional file 4: Table S2). Cortisol studies of meditation and stress reduction have produced mixed findings and lacked methodological rigor [55], rendering the present analytic strategy potentially applicable to future studies of prolonged stress. HPA-axis reactivity $\left(\mathrm{AUC}_{\mathrm{I}}\right)$ has been suggested as a potential marker of physiological reactivity to stressors, such as psychosocial stress [32]. However, cortisol is complexly related with prolonged stress and further studies of HPA-axis dynamics, prolonged stress, and burnout are clearly needed [25].

\section{Visual perception}

The threshold of conscious visual perception, $t_{0}$, improved significantly more in OC than in controls, also when adjusting for baseline. Further, larger $t_{0}$-improvements were associated with increased OC compliance. This corroborates the previously reported finding that the TVA $t_{0}$ parameter was specifically improved by meditation and not by physical stress reduction [42]. Interestingly, the TVA-model $[12,13]$ states that $t_{0} \mathrm{im}$ provements may reflect stronger reliance on bottom-updriven perception, rather than conscious recalibration of attentional weights. OC may therefore have improved the perceptual threshold because participants became less prone to consciously modulate visual attention. This aligns with the $\mathrm{OC}$ training in relaxed and receptive 
("Open") awareness of sensory information and a nonintervening ("Calm") conscious witnessing. As mentioned, these are essential elements for many meditative traditions. Correspondingly, the visual perceptual threshold was also improved by yoga $[8,78]$ and mindfulness meditation [42, 52]. Mindfulness has also improved the threshold for conscious registration of proprioceptive stimuli [62] and the perceptual threshold in an auditory temporal discrimination task [27]. As argued by recent theories, meditation may facilitate insight into personal states and promote objective perception in general through increased perceptual sensitivity within several sensory modalities, i.e., through a lowering of the stimulation needed for conscious registration [4, 14]. Our findings support these proposals, but clearly more research on bottom-up perceptual effects of meditation is needed.

\section{Experiences from the practical implementation}

The Open and Calm program received a $94 \%$ completion rate. GPs found it easy to use a simple, online referral system and maintaining the full screening at the program distributor (Copenhagen University Hospital) ensured similar inclusion procedures throughout. However, among 20 referring GPs, ten GPs referred only one-two patients each. GPs and psychiatrists are generally not accustomed to referring stressed, but otherwise healthy individuals to treatment [57]. To achieve sustainability, we reiterate recommendations [45] that mental health program distributors employ health workers specifically for sustaining recruitment through local health facilities.

The two intervention formats (individual/groups of $n=8$ ) yielded similar treatment effects (Additional file 2: Panel S1). This is important, since individual courses required 2.6 times more professional contact hours per participant. Workshops for even larger groups also reduced stress $[10,53]$ and anxiety [9]. A stepped care model [26] may be recommendable, where less intensive or demanding group programs are offered as a first-line treatment, while smaller or individual courses are offered when deemed necessary. A less intensive (minimal contact) group OC intervention is currently investigated. In general, more systematic research is needed on public health intervention formats [45]. The $\mathrm{OC}$ intervention differs from other programs mainly because it was specifically designed for public mental health promotion in a broad demographic group (A full intervention description can be supplied by request to the first author). OC was thus carried out in a health promotion clinic, not in e.g., hospital settings. OC prioritizes everyday words such as Open and Calm, rather than e.g., beginner's mind, non-judgment, or mindfulness [44]. Perhaps most importantly, OC teaches meditation as a definable strategy and not as a special state of mind (for a discussion of these contrasting approaches, see Shapiro \& Walsh [69]). OC finally emphasizes a body-psycho-social focus on promoting mental health, rather than a more narrow focus on meditation. Therefore, findings may not be generalizable to other types of meditation-based programs.

The dropout rate of only $6 \%$ may be important. Dropout in meditation-based stress reduction programs typically ranges $15-30 \%$ [64]. Based on participant feedback, the most appreciated elements of the OC program were the meditative practices and the programme structure, repeating bodily, mental, and social themes. This, however, is speculative and should be clarified by qualitative studies. We speculate that the choice of conducting evening sessions also lowered the dropout rate, especially for employed OC group participants, enabling them to maintain a normal working week. Individual OC participants could flexibly book course sessions in expanded working hours (8 am-6 pm).

Limitations of the RCT include the need for studying longer time periods, such as a year. A longer study period would enable more direct health impact assessments [49], such as measures of the occurrence of stress-related depression or days of stress-induced absence from work. An active control group would have improved the ability to detect OC-specific effects. However, an unrestricted TAU design allowed a comparison of OC with the current, unsystematic treatments offered for healthy adults dealing with prolonged stress. As another limitation, the paucity of significant associations between $\mathrm{OC}$ session attendance rates and outcome change scores should not be extrapolated to indicate that compliance with meditation is unimportant in OC or similar programs. The absence of evidence is not the evidence of absence - and several studies of short-term meditation programs did find that increased meditation compliance was related to larger treatment effects [79]. The simple compliance measure of session attendance may have prevented us from detecting such associations. More detailed compliance measures are most likely necessary to illuminate the importance of compliance with different elements of such programs. Relatedly, our relatively low sample size, especially in the cortisol analyses, limits the statistical power to detect treatment effect moderators, so these secondary findings should also be interpreted cautiously.

\section{Conclusion}

This RCT revealed that the OC program designed specifically for public stress reduction and mental health promotion improved self-reported stress, depressive symptoms, sleep disturbances, mental health, and quality of life, a physiological stress marker (the magnitude of 
cortisol secretion), and the threshold for visual perception significantly more than treatment as usual for Danish, stressed adults. The program participant completion rate was $94 \%$. All self-report effects were sustained or further improved at 3 months follow-up. We found no consistent effect moderation by age, gender, education, 5-HTTLPRgenotype, or any other control variables, while higher trait harm-avoidance might attenuate effects of OC. In sum, the OC program was consistently supported as effective. Further testing of potential advantages, including longterm more direct health sectorial benefits, of the OC program is therefore warranted. Due to the positive results, a larger implementation of the OC program is in progress in the health promotion sector in the municipality of Copenhagen.

\section{Additional files}

Additional file 1: Table S1. Sample characteristics. (DOCX 24 kb)

Additional file 2: Panel S1. Comparisons of interventional formats on self-report and visual perception. (PDF $72 \mathrm{~kb}$ )

Additional file 3: Supplementary findings: Potential effect moderation by TCl-Harm Avoidance. (DOCX $119 \mathrm{~kb}$ )

Additional file 4: Table S2. Treatment effects on cortisol and visual attention. (DOCX 29 kb)

Additional file 5: Panel S2. Open and Calm participants' changes in cortisol secretion dynamics. (PDF $60 \mathrm{~kb}$ )

\section{Competing interests}

The OC program is now implemented on a larger scale in the City of Copenhagen, which benefits the first author (CGJ), who is now partly funded as a director of this implementation. GMK has received honoraria as a consultant for $\mathrm{H}$. Lundbeck $\mathrm{A} / \mathrm{S}$, as a member of the steering group for Brain Prize. She is also on the advisory board for the Kristian G. Jebsen Foundation and a field editor for Int J Neuropsychopharm. John W. Denninger holds a position at the Benson-Henry Institute for Mind Body Medicine at Massachusetts General Hospital, which is paid by patients and their insurers for running the SMART-3RP and related relaxation/mindfulness clinical programs, markets related products such as books, DVDs, CDs and the like, and holds a patent pending (PCT/US2012/049539 filed August 3, 2012) entitled "Quantitative Genomics of the Relaxation Response." He also receives support for investigator-initiated studies from Basis/Intel and Onyx/Amgen. The other authors declared no competing interests.

\section{Authors' contributions}

CGJ conceived of the study, was the main developer behind the intervention and principal in conducting the statistical analyses and writing the manuscript. $J$ participated in designing the online intervention elements, and conducted $40 \%$ of the tests sessions, and participated in the statistical analyses of the visual test and the writing of the manuscript. AP and SAV participated in programming, analyzing, and interpreting the visual test, and in writing the manuscript. SPR conducted $60 \%$ of the test sessions and participated in coordination of participants, and in writing the manuscript. VGF headed the collection, analyses, and interpretation of cortisol data and participated in writing the manuscript. DA headed the collection, analyses, and determination of genotypical variations. GMK and SGH participated in designing the study, analyzing all types of data, and writing the manuscript, and SGH performed the randomizations. JD participated in developing the intervention, planning and supervising the study, and writing the manuscript. All authors read and approved the final manuscript.

\section{Acknowledgements}

The study was funded by Nordea-fonden, Copenhagen University Hospital, The Capital Region of Denmark, and the Lundbeck Foundation Center for Integrated Molecular Brain Imaging (Cimbi).

The authors acknowledge Tine Norup, Lisbeth Frölich, Tine Meyer Thomson, Peg Baim, Angela Krogsgaard, Freja Filine Petersen, and Pauline Voss Romme for their contribution to developing the intervention, and Peter Jensen and Agnete Dyssegaard for assistance in data handling and cortisol analyses, respectively.

\section{Author details}

${ }^{1}$ Neurobiology Research Unit (NRU) and Center for Integrated Molecular Brain Imaging (Cimbi), The Neuroscience Centre, Rigshospitalet and University of Copenhagen, Juliane Maries Vej 28, 3rd floor, 2100 Copenhagen OE, Denmark. ${ }^{2}$ Center for Visual Cognition, Department of Psychology, University of Copenhagen, Øster Farimagsgade 2A, 1353 Copenhagen K, Denmark. ${ }^{3}$ Benson-Henry Institute of Mind-Body Medicine, Massachusetts General Hospital, Boston, USA. ${ }^{4}$ Danish Dementia Center, Copenhagen University Hospital, Copenhagen, Denmark.

Received: 6 January 2015 Accepted: 9 December 2015

Published online: 16 December 2015

\section{References}

1. Aiken LS, West SG. Multiple regression: testing an interpreting interactions. Newbury Park, CA: Sage; 1991.

2. Anderson N, Johnson S, Belar C, Breckler S, Nordal K, Ballard D. Stress in America: Our Health at Risk. Washington, D.C.: American Psychological Association; 2012.

3. Bech P, Rasmussen NA, Olsen LR, Noerholm V, Abildgaard W. The sensitivity and specificity of the Major Depression Inventory using the present state examination as the index of diagnostic validity. J Affect Disord. 2001;66:159-64.

4. Bedford FL. A perception theory in mind-body medicine: guided imagery and mindful meditation as cross-modal adaptation. Psychon Bull Rev. 2012; 19:24-45.

5. Bjørner JB, Damsgaard MT, Watt T, Bech P, Rasmussen, Thunedborg K Dansk manual til SF-36. København: Lif; 1997.

6. Booij SH, Bouma EMC, de Jonge P, Ormel J, Oldehinkel AJ. Chronicity of depressive problems and the cortisol response to psychosocial stress in adolescents: the TRAILS study. Psychoneuroendocrinology. 2013;38(5):659-65

7. Borg V, Nexø MA, Kolte IV, Andersen MF. Hvidbog om mentalt helbred, sygefravær og tilbagevenden til arbejde. Copenhagen: Nationalt Forskningscenter for Arbejdsmiljø; 2010.

8. Braboszcz C, Cahn BR, Balakrishnan B, Maturi RK, Grandchamp R, Delorme A. Plasticity of visual attention in Isha yoga meditation practitioners before and after a 3-month retreat. Front Psychol. 2013;4:914-914.

9. Brown JS, Cochrane R, Mack C, Leung N, Hancox T. Comparison of effectiveness of large scale stress workshops with small stress/anxiety management training groups. Behav Cogn Psychother. 1998;26:219-35.

10. Brown JSL, Cochrane R, Hancox T. Large-scale health promotion stress workshops for the general public: a controlled evaluation. Stress. 2000;28:139-51.

11. Brown KW, Ryan RM. The benefits of being present: mindfulness and its role in psychological well-being. J Pers Soc Psychol. 2003;84:822-48.

12. Bundesen C. A theory of visual attention. Psychol Rev. 1990;97:523-47.

13. Bundesen $C$, Habekost T, Kyllingsbaek S. A neural theory of visual attention: bridging cognition and neurophysiology. Psychol Rev. 2005;112:291-328.

14. Bushell WC. New beginnings: evidence that the meditational regimen can lead to optimization of perception, attention, cognition, and other functions. Ann N Y Acad Sci. 2009;1172:348-61.

15. Buysse DJ, Reynolds CF, Monk TH, Berman SR, Kupfer DJ. The Pittsburgh sleep quality index: a new instrument for psychiatric practice and research. Psychiatry Res. 1989;28:193-213.

16. Canli T, Lesch K-P. Long story short: the serotonin transporter in emotion regulation and social cognition. Nat Neurosci. 2007;10:1103-9.

17. Carmody J, Baer RA. How long does a mindfulness-based stress reduction program need to be? a review of class contact hours and effect sizes for psychological distress. J Clin Psychol. 2009;65:627-38. 
18. Chang B-H, Casey A, Dusek JA, Benson H. Relaxation response and spirituality: pathways to improve psychological outcomes in cardiac rehabilitation. J Psychosom Res. 2010;69(2):93-100.

19. Charney DS. Psychobiological mechanisms of resilience and vulnerability: implications for successful adaptation to extreme stress. Am J Psychiatry. 2004;161:195-216.

20. Chen MC, Hallmayer J, Joormann J, Gotlib IH. Serotonin transporter polymorphism predicts waking cortisol in young girls. Psychoneuroendocrinology. 2009;34:681-6.

21. Chiesa A, Serretti A. Mindfulness-based stress reduction for stress management in healthy people: a review and meta-analysis. J Altern Complement Med. 2009;15:593-600.

22. Christensen Al, Ekholm O, Davisen M, Juel K. Sundhed og sygelighed i Danmark 2010 - og udviklingen siden 1987. Copenhagen: The Danish National Institute of Public Health; 2012.

23. Cloninger CR, Przybeck TR, Svrakic DM. The Temperament and Character Inventory (TCI): A guide to its development and use. St. Louis: Washington University; 1994

24. Cohen S, Williamson G. In: Spacapam S, Oskamp S, editors. Perceived stress in a probability sample of the United States. CA: Sage. Newbury Park; 1988.

25. Danhof-Pont MB, Van Veen T, Zitman FG. Biomarkers in burnout: a systematic review. J Psychosom Res. 2011;70:505-24.

26. Davison GC. Stepped care: doing more with less? J Consult Clin Psychol. 2000;68:580

27. Droit-Volet S, Fanget M, Dambrun M. Mindfulness meditation and relaxation training increases time sensitivity. Conscious Cogn. 2015;31:86-97.

28. Dyrholm M, Kyllingsbæk S, Espeseth T, Bundesen C. Generalizing parametric models by introducing trial-by-trial parameter variability: the case of TVA. J Math Psychol. 2011;55:416-29.

29. Faul F, Erdfelder E, Lang A, Buchner A. G*Power 3: A flexible statistical power analysis program for the social, behavioral, and biomedical sciences. Behav Res Methods. 2007:39:175-91.

30. Fekedulegn DB, Hartley TA, Andrew ME, Burchfiel CM, Violanti JM, Miller DB. Area under the curve and other summary indicators of repeated waking cortisol measurements. Psychosom Med. 2007;69:651-9.

31. Folker $\mathrm{H}$, Folker AP. WHO-5 as a simple method for measuring quality of life in daily psychiatric clinics. [Article in Danish]. Ugeskr Laeger. 2008;170:830-4.

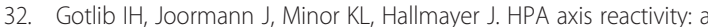
mechanism underlying the associations among 5- HTTLPR, stress, and depression. Biol Psychiatry. 2008;63:847-51.

33. Goyal M, Singh S, Sibinga EMS, Gould NF, Rowland-Seymour A, Haythornthwaite JA. Meditation programs for psychological stress and well-being: a systematic review and meta-analysis. JAMA Intern Med. 2014; 174:357-68.

34. Grossman P, Niemann L, Schmidt S, Walach H. Mindfulness-based stress reduction and health benefits: a meta-analysis. J Psychosom Res. 2004:57:35-43

35. Gu J, Strauss C, Bond R, Cavanagh K. How do mindfulness-based cognitive therapy and mindfulness-based stress reduction improve mental health andWellbeing? a systematic review and meta-analysis of mediation studies. Clin Psychol Rev. 2015;37:1-12.

36. Gulis G, Eriksen ML, Aro AR. Public health research in Denmark in the years 1995-2005. Scand J Public Health. 2010:38(1):104-7.

37. Hill MN, Hellemans KG, Verma P, Gorzalka BB, Weinberg J. Neurobiology of chronic mild stress: parallels to major depression. Neurosci Biobehav Rev. 2012;36:2085-117

38. Jakobsen P, Bach E, Pedersen JM, Thorsen SV, Thomsen BL, Christensen BS. Arbejdsmiljø og helbred i Danmark, 2012 [Working environment and health in Denmark, 2012]. Copenhagen: National Research Centre for the Working Environment; 2013.

39. Jensen CG. Åben og Rolig [Open and Calm]. Copenhagen: Strandberg Publishing; 2013

40. Jensen, CG. Åben og Rolig - Standardiseret Instruktørmanual [Open and Calm - Standardized Instructor's Manual]. Copenhagen: Centre for Mental Health Promotion. 2015. (www.cfps.dk).

41. Jensen CG, Nicklasen J, Petersen A, Vangkilde S, Hasselbalch SG. General Inattentiveness is a Long-term Reliable Trait Independently Predicative of Psychological Health: Danish Validation Studies of Mindfulness Attention Awareness Scale, Psychological Assessment. In press.

42. Jensen CG, Vangkilde S, Frokjaer V, Hasselbalch SG. Mindfulness training affects attention - or is it attentional effort? J Exp Psychol Gen. 2012;141:106-23.
43. Juster R-P, Sindi S, Marin M-F, Perna A, Hashemi A, Pruessner JC, et al. A clinical allostatic load index is associated with burnout symptoms and hypocortisolemic profiles in healthy workers. Psychoneuroendocrinology. 2011:36:797-805.

44. Kabat-Zinn J. Wherever you go, there you are: mindfulness meditation in everyday life. New York: Hyperion; 1994.

45. Kalra G, Christodoulou G, Jenkins R, Tsipas V, Christodoulou N, Lecic-Tosevski $D$, et al. Mental health promotion: guidance and strategies. Eur Psychiatry. 2012;27:81-6.

46. Karg K, Burmeister M, Shedden K, Sen S. The serotonin transporter promoter variant (5-HTTLPR), stress, and depression meta-analysis revisited: evidence of genetic moderation. Arch Gen Psychiatry. 2011;68:444-54.

47. Kendler KS, Kessler RC, Walters EE, Maclean C, Neale MC, Heath AC, et al. Stressful life events, genetic liability, and onset of an episode of major depression in women. Am J Psychiatry. 1995;152:833-42.

48. Keng S-L, Smoski MJ, Robins CJ. Effects of mindfulness on psychological health: a review of empirical studies. Clin Psychol Rev. 2011;31(6):1041-56.

49. Kraemer SRJ, Gulis G. How do experts define relevance criteria when initiating Health Impact Assessments of national policies? Scand J Public Health. 2014;42(1):18-24.

50. Kraemer HC, Wilson GT, Fairburn CG, Agras WS. Mediators and moderators of treatment effects in randomized clinical trials. Arch Gen Psychiatry. 2002;59(10):877-83.

51. Lucassen PJ, Pruessner J, Sousa N, Almeida OFX, Van Dam AM, Rajkowska G, et al. Neuropathology of stress. Acta Neuropathol. 2014;127:109-35.

52. Maclean KA, Ferrer E, Aichele SR, Bridwell DA, Zanesco AP, Saron CD. Intensive meditation training improves perceptual discrimination and sustained attention. Psychol Sci. 2010;21:829-39.

53. Main NA, Elliot SA, Brown JSL. Comparison of three different approaches used in large-scale stress workshops for the general public. Behav Cogn Psychother. 2005;33:299-309.

54. Marchand A, Juster R-P, Durand P, Lupien SJ. Burnout symptom sub-types and cortisol profiles: What's burning most? Psychoneuroendocrinology. 2014:40:27-36

55. Matousek RH, Dobkin PL, Pruessner J. Cortisol as a marker for improvement in mindfulness-based stress reduction. Complement Ther Clin Pract. 2010;16:13-9.

56. McEwen BS. Brain on stress: how the social environment gets under the skin. Proc Natl Acad Sci U S A. 2012;109(Suppl):17180-5.

57. Min JA, Lee CU, Lee C. Mental Health Promotion and Illness Prevention: A Challenge for Psychiatrists. Psychiatry Investigation. 2013;10(4):307-316. http://doi.org/10.4306/pi.2013.10.4.307.

58. Morris SB, Deshon RP. Combining effect size estimates in meta-analysis with repeated measures and independent-groups designs. Psychol Methods. 2002;7:105-25.

59. Moya-Albiol L, Serrano M, Salvador A. Job satisfaction and cortisol awakening response in teachers scoring high and low on burnout. Span J Psychol. 2010;13:629-36.

60. Mueller A, Brocke B, Fries E, Lesch K-P, Kirschbaum C. The role of the serotonin transporter polymorphism for the endocrine stress response in newborns. Psychoneuroendocrinology. 2010;35(2):289-96.

61. Murakami H, Matsunaga M, Ohira H. Association of serotonin transporter gene polymorphism and emotion regulation. Neuroreport. 2009;20(4):414-8. http://doi.org/10.1097/WNR.0b013e328325a910.

62. Naranjo JR, Schmidt S. Is it me or not me? Modulation of perceptual-motor awareness and visuomotor performance by mindfulness meditation. BMC Neurosci. 2012;13:88

63. Olsen LR, Mortensen EL, Bech P. Prevalence of major depression and stress indicators in the Danish general population. Acta Psychiatr Scand. 2004;109:96-103.

64. Ospina MB, Bond K, Karkhaneh M, Tjosvold L, Vandermeer B, Klassen TP. Meditation practices for health: state of the research. Evid Rep Technol Assess. 2007;155:1-263.

65. Park ER, Traeger L, Vranceanu A, Scult M, Lerner J, Fricchione G. The development of a patient-centered program based on the relaxation response: the Relaxation Response Resiliency Program (3RP). Psychosomatics. 2013;54:165-74.

66. Porkka-Heiskanen T, Zitting KM, Wigren HK. Sleep, its regulation and possible mechanisms of sleep disturbances. Acta Physiol (Oxf). 2013;208:311-28.

67. Pruessner JC, Hellhammer DH, Kirschbaum C. Burnout, perceived stress, and cortisol responses to awakening. Psychosom Med. 1999;61:197-204. 
68. Sedlmeier P, Eberth J, Schwarz M, Zimmermann D, Haarig F, Jaeger S, et al. The psychological effects of meditation: a meta-analysis. Psychol Bull. 2012;138:1139-71.

69. Shapiro DH, Walsh RN. Meditation: Classical and contemporary perspectives. New York: Aldine; 1984.

70. Statistics Denmark (2014). Education table: area: Copenhagen; year: 2013; age groups 20-59 years. Retrieved Nov. 11, 2014: http://www.statistikbanken. $\mathrm{dk} / \mathrm{KRHFU} 2$

71. Stigsdotter UK, Ekholm O, Schipperijn J, Toftager M, Kamper-Jørgensen F, Randrup TB. Health promoting outdoor environments-associations between green space, and health, health-related quality of life and stress based on a Danish national representative survey. Scand J Public Health. 2010;38:411-7.

72. Stoyanov DS, Cloninger CR. Relation of people-centered public health and person-centered healthcare management: a case study to reduce burn-out. Int J Pers Cent Med. 2012;2:90.

73. Tabachnick BG, Fidell LS. Using multivariate statistics. 5th ed. Boston: Pearson International; 2007.

74. Toneatto T, Nguyen L. Does mindfulness meditation improve anxiety and mood symptoms? A review of the controlled research. Canadian Journal of Psychiatry. Revue Canadienne de Psychiatrie. 2007;52(4):260-266. Retrieved from http://www.ncbi.nlm.nih.gov/pubmed/17500308.

75. Uher R, McGuffin P. The moderation by the serotonin transporter gene of environmental adversity in the aetiology of mental illness: review and methodological analysis. Mol Psychiatry. 2008;13(2):131-46.

76. Van Breukelen GJP, Van Dijk KRA. Use of covariates in randomized controlled trials. J Int Neuropsychol Soc. 2007;13(5):903-4.

77. Vangkilde S, Bundesen C, Coull JT. Prompt but inefficient: nicotine differentially modulates discrete components of attention. Psychopharmacology (Berl). 2011;218:667-80

78. Vani PR, Nagarathna R, Nagendra HR, Telles S. Progressive increase in critical flicker fusion frequency following yoga training. Indian J Physiol Pharmacol. 1997:41:71-4.

79. Vettese LC, Toneatto T, Stea JN, Nguyen L, Wang JJ. Do Mindfulness Meditation Participants Do Their Homework? And Does It Make a Difference? A Review of the Empirical Evidence. Journal of Cognitive Psychotherapy. 2009; 23(3):198-225. http://doi.org/10.1891/0889-8391.23.3.198.

80. Vickers AJ. How many repeated measures in repeated measures designs? Statistical issues for comparative trials. BMC Med Res Methodol. 2003;3:22.

81. Virgili M. Mindfulness-based interventions reduce psychological distress in working adults: a meta-analysis of intervention studies. Mindfulness. 2013;6:326-37.

82. Ware JE, Snow KK, Kosinski M, Gandek B. SF-36 Health Survey. Manual and Interpretation Guide. Boston, MA: The Health Institute, New England Medical Center; 1993.

83. Wendland JR, Martin BJ, Kruse MR, Lesch KP, Murphy DL. Simultaneous genotyping of four functional loci of human SLC6A4, with a reappraisal of 5-HTTLPR and rs25531. Mol Psychiatry. 2006;11:224-6.

84. World Health Organization. Mental health: facing the challenges, building solutions: report from the WHO European Ministerial Conference. Copenhagen: WHO; 2005. ISBN 92-890-1377-X.

\section{Submit your next manuscript to BioMed Central and we will help you at every step:}

- We accept pre-submission inquiries

- Our selector tool helps you to find the most relevant journal

- We provide round the clock customer support

- Convenient online submission

- Thorough peer review

- Inclusion in PubMed and all major indexing services

- Maximum visibility for your research

Submit your manuscript at www.biomedcentral.com/submit 